\title{
INFLUENCE OF EXCITATION PROCESSES ON THE SHAPE OF ARGON AND NEON LINES
}

\author{
A. Bielski, R. Ciurylo, D. Lisak and R.S. Trawiński \\ Institute of Physics, Nicholas Copernicus University \\ Grudziądzka 5/7, 87-100 Toruń, Poland
}

(Received September 21, 1999; revised version November 30, 1999)

Shapes of self-broadened argon lines $687.1 \mathrm{~nm}\left(4 d_{5}-2 p_{10}\right), 703.0 \mathrm{~nm}$ $\left(3 s_{5}-2 p_{9}\right), 750.3 \mathrm{~nm}\left(2 p_{1}-1 s_{2}\right)$ as well as the neon line $748.8 \mathrm{~nm}\left(3 d_{3}-2 p_{10}\right)$ emitted from glow discharges at low pressures were analysed using a FabryPerot interferometer. We showed that the dissociative recombination of $\mathrm{Ar}_{2}^{+}$ (or $\mathrm{Ne}_{2}^{+}$) molecular ions which gives rise to the density of non-thermalized atoms, may be regarded as the main process responsible for the distortion of the profiles of the above lines. The distorted profiles were analysed as superposition of the Ballik profile for thermalized atoms and the modified Ballik profile for non-thermalized atoms. The Lorentzian and Gaussian widths as well as relative densities of non-thermalized and thermalized atoms were determined.

PACS numbers: $32.70 .-\mathrm{n}, 33.70 .-\mathrm{w}, 34.20 .-\mathrm{b}$

\section{Introduction}

Experiments on self-broadening of several neon lines corresponding to the transition array $\left(2 p^{5} 3 d-2 p^{5} 3 p\right)$ emitted from the glow discharge at pressures about 1 Torr have revealed systematic departures of the observed profiles from Voigt profiles $[1,2]$. They were satisfactorily explained [2] as caused by dissociative recombination of molecular ions $\mathrm{Ne}_{2}^{+}$with electrons. Up to now there were no observations of similar departures for argon lines emitted under above conditions. Frommhold and Biondi [3] have suggested, however, that also in the case of argon the process of dissociative recombination of molecular $\mathrm{Ar}_{2}^{+}$ions may influence the observed line shape.

In recent years a systematic study of the shapes of self-broadened argon lines $687.1 \mathrm{~nm}\left(4 d_{5}-2 p_{10}\right), 703.0 \mathrm{~nm}\left(3 s_{5}-2 p_{9}\right), 750.3 \mathrm{~nm}\left(2 p_{1}-1 s_{2}\right)$ as well as neon line $748.8 \mathrm{~nm}\left(3 d_{3}-2 p_{10}\right)$ emitted from low-current glow discharge sources has been performed in our laboratory using a pressure-scanned Fabry-Perot interferometer [4-7]. For the $687.1 \mathrm{~nm}$ and $703.0 \mathrm{~nm}$ Ar lines and the $748.8 \mathrm{~nm}$ Ne line we have found that at pressures below 3 Torr the observed profiles differ significantly 
from the Voigt function. Following Stacey and Thompson [1] such departures may be explained as due to effects associated with mechanism by which the emitting atoms are excited. In the present paper we focus our attention on the dissociative recombination of molecular ions $\mathrm{Rg}_{2}^{+}(\mathrm{Rg}=\mathrm{Ar}, \mathrm{Ne})$ with electrons which can cause incomplete thermalization of the excited $\mathrm{Rg}$ atoms [3, 8].

The dissociative recombination of $\mathrm{Rg}_{2}^{+}$ions with electrons can be described by the reaction

$$
\mathrm{Rg}_{2}^{+}+\mathrm{e} \longrightarrow\left(\mathrm{Rg}_{2}^{*}\right)_{\text {unstable }} \longrightarrow \mathrm{Rg}^{*}+\mathrm{Rg}+\text { kinetic energy }
$$

where the superscripts ${ }^{+}$and ${ }^{*}$ refer to ionized and excited states, respectively. In this reaction a molecular rare-gas ion captures an electron to form an unstable excited molecule which then begins to dissociate producing two atoms, one in an excited state, moving apart with kinetic energies prevailing the kinetic energy corresponding to the gas temperature. In an earlier paper [2] this reaction was adapted as a basis for quantitative description of departures of the observed profiles of the $753.5 \mathrm{~nm}$ and $754.4 \mathrm{~nm}$ neon lines from the Voigt profile. The main goal of the present work was to apply this model to analyse the profiles of three lines of argon (687.1, 703.0, and $750.3 \mathrm{~nm}$ ) as well as the $748.8 \mathrm{~nm}$ line of neon where for the first two argon lines and for the neon line distortion of the profiles were observed.

The process of dissociative recombination in argon which is schematically represented in Fig. 1 and Eq. (1) has been thoroughly studied by Biondi and his

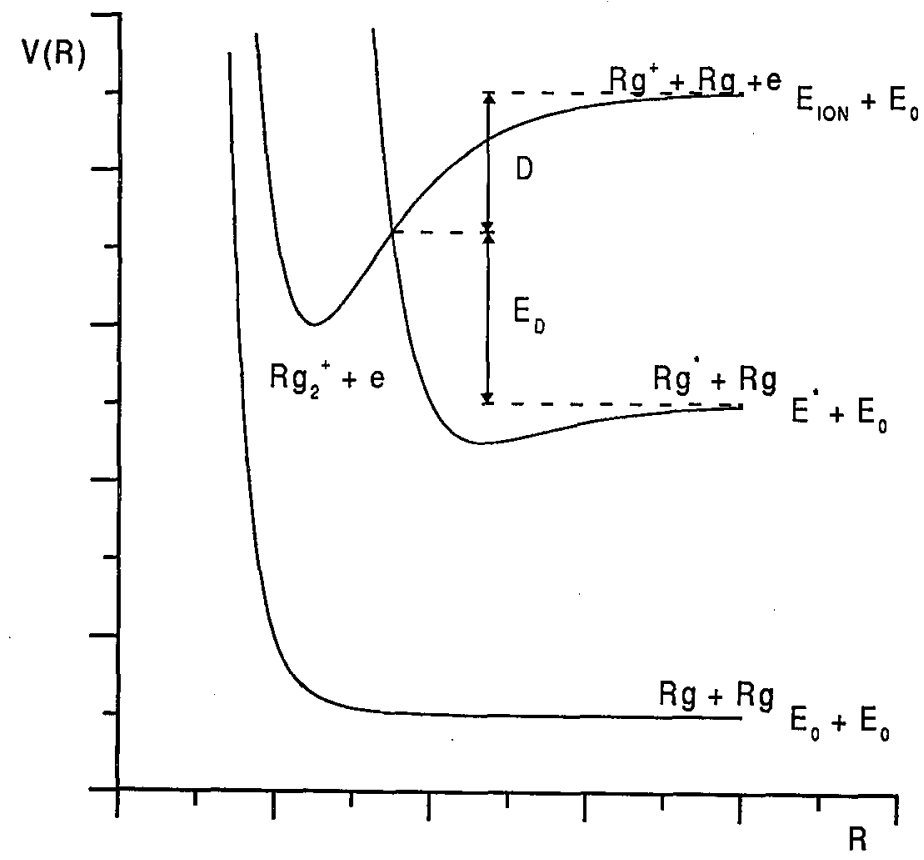

Fig. 1. The scheme of dissociative recombination process in rare gases. Notations explained in the text. 
coworkers $[3,8-10]$ who performed high-resolution studies of line profiles to detect excess kinetic energy of dissociation fragments.

Connor and Biondi [8] and Frommhold and Biondi [3] have performed interferometric measurements of the profiles of many neon and argon lines emitted from a microwave discharge and during the ensuing afterglow. They have found that in the afterglow the Doppler profiles are much broader than the thermal atom profiles of the lines observed in the discharge. Moreover, this excess Doppler broadening of lines in the afterglow phase was found to decrease with increasing neon (argon) pressure which may be interpreted as due to thermalizing collisions.

\section{Spectral line shapes for thermalized excited atoms}

Under the conditions of experiments performed in our laboratory on argon (or neon) spectral. lines emitted from glow discharge sources, it is expected that the plasma is created by electron impact excitation and ionization of ground-state atoms. The atomic $\mathrm{Rg}^{+}$ions are formed directly, and the molecular $\mathrm{Rg}_{2}^{+}$ions are produced due to associative ionization [11] when highly excited argon (or neon) atoms combine with ground-state atoms.

In our previous works on argon line shapes $[4,5]$ we have assumed that all excited argon atoms are thermalized and have the same velocity distribution as the ground-state atoms. Such an assumption is usually justified in case of glow discharges.

Assuming the kinetic energy distribution of the excited atoms to be Maxwellian at the gas temperature $T$, the Doppler line shape $I_{D}(\omega)$ corresponding to the motion of the thermalized excited argon atoms can be represented by a Gaussian distribution with the halfwidth (FWHM) given by

$$
\gamma_{\mathrm{D}}=2 \frac{\omega_{0}}{c} \sqrt{\frac{2 k T}{m} \ln 2}
$$

where $\omega_{0}$ denotes the unperturbed frequency of the line, and $c$ is the speed of light, $k$ is the Boltzmann constant and $m$ is the mass of the emitting atom.

At low gas densities the intensity distribution due to pressure broadening is given by the Lorentzian distribution $I_{L}(\omega)$ characterized by the halfwidth (FWHM) $\gamma_{\mathrm{L}}$ and the shift $\Delta$ which are linearly dependent on the number density $N$ of the gas.

Let $A(\omega)$ denote the instrumental profile. If we ignore any correlations between the Doppler and pressure broadening (which for self-broadening of argon and neon lines in the pressure range used in this work is a sufficient approximation) then the resultant intensity distribution $I_{\text {th }}(\omega)$ of a spectral line emitted by thermalized excited $\mathrm{Rg}$ atoms may be written as a convolution

$$
I_{\mathrm{th}}(\omega)=A(\omega) \otimes I_{\mathrm{L}}(\omega) \otimes I_{\mathrm{D}}(\omega)=A(\omega) \otimes F(\omega),
$$

where $F(\omega)$ is the Voigt profile.

In the case of the Fabry-Perot interferometer the instrumental profile $A(\omega)$ may be expressed by a well-known Airy formula (cf. e.g. [12]). Ballik [13] has shown that in such a case, Eq. (3) which represents a convolution of the Airy distribution with the Voigt profile, may be written as

$$
I_{\mathrm{th}}(\omega)=\frac{1}{\Omega}\left\{\frac{1}{2}+\sum_{n=1}^{\infty} R^{n} \mathrm{e}^{-n L} \mathrm{e}^{-n^{2} D^{2} / 4} \cos \left[\frac{2 n \pi}{\Omega}\left(\omega-\omega_{0}-\Delta\right)\right]\right\},
$$


where $L=\pi \gamma_{\mathrm{L}} / \Omega$ and $D=\pi \gamma_{\mathrm{D}} /(\Omega \sqrt{\ln 2}), \Omega$ is the free spectral range, and $R$ is the reflection coefficient of the coatings of the Fabry-Perot interferometer plates.

\section{Dissociative recombination and the combined Doppler and pressure broadening}

In the model described in [2] the dissociative recombination is assumed to be the main process responsible for the occurrence of non-thermalized excited atoms. It was shown that the resultant profile $I_{\text {ntll }}(\omega)$ of a line emitted by non-thermalized excited atoms, i.e. those atoms which are produced due to dissociative recombination process, can be written in the following way:

$I_{\mathrm{nth}}(\omega)=\frac{1}{\Omega}\left\{\frac{1}{2}+\sum_{n=1}^{\infty} R^{n} \mathrm{e}^{-n L} \mathrm{e}^{-n^{2} D^{2} / 8} \operatorname{Sinc}(n B) \cos \left[\frac{2 n \pi}{\Omega}\left(\omega-\omega_{0}-\Delta\right)\right]\right\}$,

where $B=\pi \gamma_{\mathrm{DIS}} / \Omega, \operatorname{Sinc}(x)=\sin (x) / x, \gamma_{\mathrm{DIS}}=2 \omega_{0} \sqrt{E_{\mathrm{D}} / m}$ with $E_{\mathrm{D}}=$ $E_{\mathrm{i}}-E^{*}-D$. Here $E_{\mathrm{i}}$ is the ionization energy of the $\mathrm{Rg}$ atom, $E^{*}$ is the energy of a given excited state, and $D$ is the energy for which the crossing of potential curves of the $\mathrm{Rg}^{*}-\mathrm{Rg}$ and $\mathrm{Rg}^{+}-\mathrm{Rg}$ systems occurs (see Fig. 1). Equation (5) will be referred to as the modified Ballik profile.

The total profile $I(\omega)$ constructed from thermalized and non-thermalized profiles can thus be written as

$$
I(\omega)=\eta_{\mathrm{th}} I_{\mathrm{th}}(\omega)+\eta_{\mathrm{nth}} I_{\mathrm{nth}}(\omega)=\frac{1}{1+\kappa}\left[I_{\mathrm{th}}(\omega)+\kappa I_{\mathrm{nth}}(\omega)\right],
$$

with $\kappa=N_{\text {nth }} / N_{\text {th }}$. Here $N_{\text {th }}$ and $N_{\text {nth }}$ denote the number densities of the thermalized and non-thermalized excited atoms, respectively. The population fraction in each case is $\eta_{\text {nth }}=N_{\text {nth }} / N_{0}$ and $\eta_{\text {th }}=N_{\text {th }} / N_{0}$, where $N_{0}=N_{\text {nth }}+N_{\text {th }}$.

It is seen that the total profile consists of two components: a thermal component which can be expressed in terms of the Ballik formula, Eq. (4), and a broader non-thermal component of the form given by the modified Ballik formula, Eq. (5).

\section{Experimental setup and results}

The argon and neon lines were formed in emission in a glow discharge source. The source was identical to that described previously [14]. The light was collected from the positive column of the $1.5 \mathrm{~mA}$ glow discharge, from a capillary of diameter $2 \mathrm{~mm}$ and length $25 \mathrm{~mm}$. Line profiles were recorded using a pressure-scanned Fabry-Perot interferometer of the type described previously [15] with dielectric coating and $1.513 \mathrm{~cm}$ spacer (free spectral range $0.3305 \mathrm{~cm}^{-1}$ ), so the line profiles were registered as the function of wave number $\widetilde{\nu}$, therefore hereafter the angular frequency $\omega$ is replaced by $\widetilde{\nu}$. The intensity distribution within a line was registered using a photomultiplier in the photon counting mode.

In order to show the role of the excitation processes on the line shape we have fitted both $I_{\text {th }}(\widetilde{\nu})$ (Eq. (4)) as well as $I(\widetilde{\nu})($ Eq. (6)) profiles to a measured profile. These profiles were fitted to a measured profile using a least-squares algorithm given by Marquardt [16].

In case of $I_{\text {th }}(\widetilde{\nu})$ the fitting parameters were $\gamma_{\mathrm{D}}, \gamma_{\mathrm{L}}$, and $\Delta$. In case of $I(\tilde{\nu})$ the fitting parameters were both $\gamma_{\mathrm{D}}, \gamma_{\mathrm{L}}, \Delta$, and $\gamma_{\mathrm{DIS}}$, and $\kappa$. Since the position 
of the maximum of the line under investigation is determined with respect to the maximum of a reference line the shift $\Delta$ may be written as

$$
\Delta=\Delta_{0}+\delta N \text {. }
$$

Here $\delta$ is the pressure-shift coefficient and $\Delta_{0}$ is a residual shift which depends, first of all, on the conditions in the reference source. We have verified that the above linear dependence of the shift on the gas density is very well fulfilled for both argon and neon lines.

In order to examine the quality of the fit we used residues in the form of the weighted differences of the intensities

$$
D_{\sigma}(\widetilde{\nu})=\frac{I_{\exp }(\widetilde{\nu})-I_{\text {fit }}(\widetilde{\nu})}{\sqrt{I_{\text {fit }}(\widetilde{\nu})}}
$$

between experimental $I_{\exp }(\widetilde{\nu})$ and theoretical (fitted) $I_{\text {fit }}(\widetilde{\nu})$ profiles. Because the experimental profiles were registered using a photomultiplier in the photon counting mode we assumed that the mean standard deviation corresponding to the best fit value at wave number $\widetilde{\nu}$ is equal to $\sqrt{I_{\text {fit }}(\widetilde{\nu})}$.

Figure 2A shows the shape of the self-broadened Ar $687.1 \mathrm{~nm}$ line measured at the pressure 0.61 Torr. In Fig. $2 \mathrm{~B}$ we plotted these residues for the case when

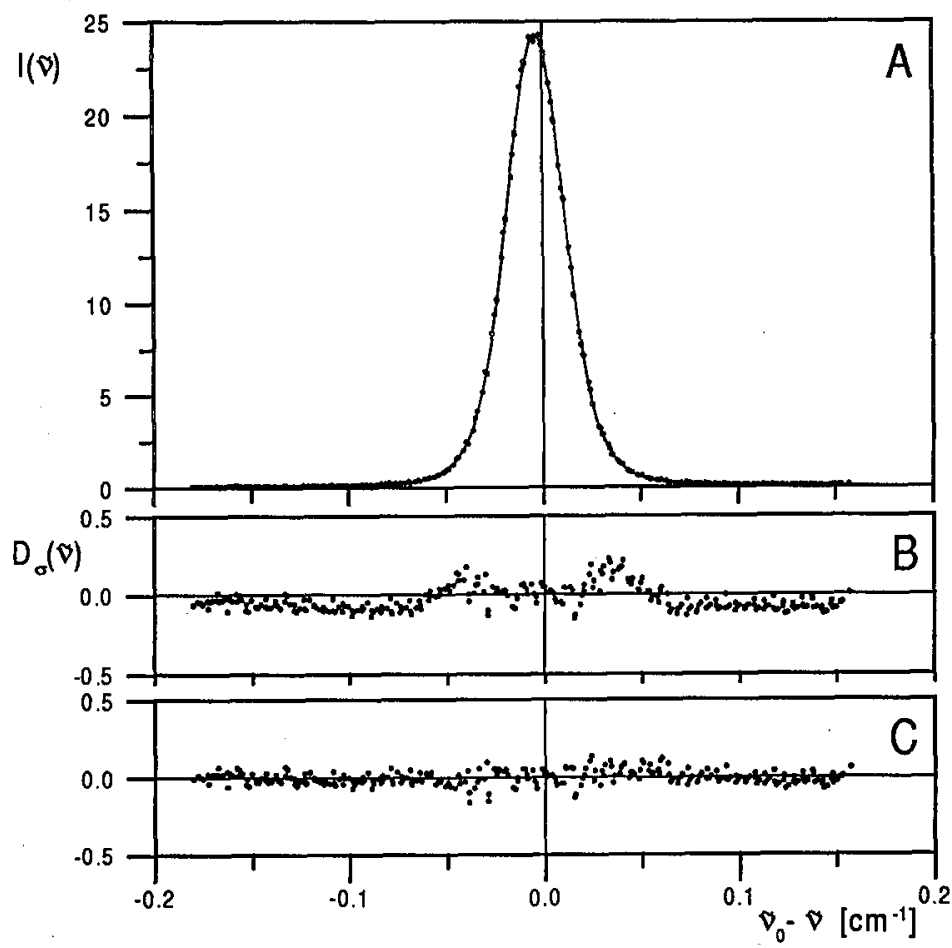

Fig. 2. (A) The shape $I(\widetilde{\nu})$ (in relative units) of the self-broadened Ar $687.1 \mathrm{~nm}$ line at pressure 0.61 Torr. (B) The weighted differences $D_{\sigma}$ (in relative units) between the experimental and the best-fit value for $I_{\mathrm{fit}}(\widetilde{\nu})=I_{\mathrm{th}}(\widetilde{\nu})$ and $(\mathrm{C})$ for $I_{\mathrm{fit}}(\tilde{\nu})=I(\widetilde{\nu})$. 
$I_{\text {fit }}(\widetilde{\nu})=I_{\text {th }}(\widetilde{\nu})$ (Eq. (4)). We can see systematic departures from zero which are similar to those observed in our earlier paper [2] for 753.5 and $754.4 \mathrm{~nm}$ neon lines. Figure $2 \mathrm{C}$ shows the residues for the case when $I_{\text {fit }}(\widetilde{\nu})=I(\widetilde{\nu})$ (Eq. (6)). As can be seen in this case the weighted differences are spread uniformly about zero which confirms the good quality of the fit.

Figures $3 \mathrm{~A}$ and $3 \mathrm{~B}$ show the best-fit values of the parameters $\kappa=N_{\text {nth }} / N_{\text {th }}$ and $\gamma_{\mathrm{DIS}}$, respectively, plotted against the argon pressure. The dependence shown in Fig. 3A is rather surprising as the ratio $\kappa$ of the total intensity of the radiation from dissociatively produced non-thermalized atoms to that of the radiation from thermalized atoms tends to increase with the increase in the argon pressure. This strange behaviour cannot be explained using such a simple description of discharge processes as applied in papers $[2,3]$ in which the rates of dissociative recombination and other excitation processes as well as the relaxation time in excited state are assumed to be independent of pressure. It is possible that the dependence shown in Fig. 3A is caused by the fact that our line shape analysis was performed on the basis of Eqs. (3) and (4). These equations are valid provided that the pressure and Doppler broadenings are statistically independent. This means that $\gamma_{\mathrm{L}}$ and $\Delta$ do not depend on the velocity of the emitting atom. In such a case the Voigt profile, i.e. the convolution of the Lorentzian and Gaussian profile, represents a good approximation to the resultant profile. This approximation fails if the pressure broadening and thermal motion of emitters are correlated. In such a case a speed-dependent Voigt profile [17] should be used instead of ordinary Voigt profile. Unfortunately for the argon line $687.1 \mathrm{~nm}$ the speed-dependent Voigt profile cannot be calculated because the real interaction potentials are not known.

Figure 3B shows that the value of $\gamma_{\mathrm{DIS}}$ is independent of the argon pressure which agrees with theoretical predictions $[2,3]$. Using the best fit value of

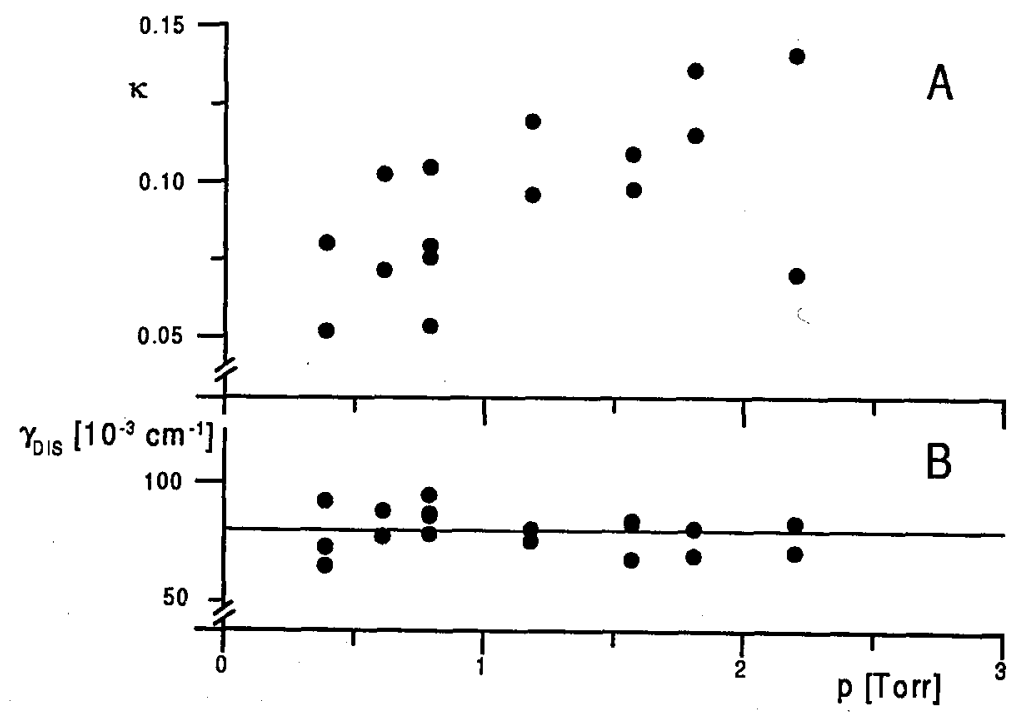

Fig. 3. The best-fit values of the parameters for Ar $687.1 \mathrm{~nm}$ line: (A) $\kappa=N_{\mathrm{nth}} / N_{\mathrm{th}}$ and (B) $\gamma_{D I S}$ plotted against the argon pressure. 
$\gamma_{\text {DIS }}=(80 \pm 9) \times 10^{-3} \mathrm{~cm}^{-1}$ determined in the present work we have found the value $E_{\mathrm{D}}$ of the kinetic energy of argon atom in excess of the kinetic energy of the thermal motion to be $E_{\mathrm{D}}=(0.28 \pm 0.06) \mathrm{eV}$. From this value we finally determined the value $D$ of the energy for which the crossing of potential curves of the $\mathrm{Ar}^{*}-\mathrm{Ar}$ and $\mathrm{Ar}^{+}-\mathrm{Ar}$ systems occurs to be $D=(0.77 \pm 0.06) \mathrm{eV}$. It should be noted that this value of $D$ fulfils the condition $D \leq D_{0}$, where $D_{0}=(1.320 \pm 0.005) \mathrm{eV}[17]$ is the dissociation energy of the molecular ion $\mathrm{Ar}_{2}^{+}$in its ground electronic state ${ }^{2} \Sigma_{\mathrm{u}}^{+}$.

The weighted differences obtained for self-broadened $703.0 \mathrm{~nm}$ Ar line at argon pressure about 1 Torr for the cases when $I_{\text {fit }}(\widetilde{\nu})=I_{\text {th }}(\widetilde{\nu})$ as well as for $I_{\text {fit }}(\widetilde{\nu})=I(\widetilde{\nu})$ are very close to those shown in Fig. 2 for the $687.1 \mathrm{~nm}$ Ar line. The dependence of the $\kappa$ parameter on the argon pressure for the $703.0 \mathrm{~nm} \mathrm{Ar}$ line has a typical decaying character, the same as observed by Biondi [8] for the afterglow and for the glow discharge [2] in neon. Using the same procedure as for the $687.1 \mathrm{~nm}$ Ar line we determined $\gamma_{\mathrm{DIS}}, E_{\mathrm{D}}$, and $D$; their values are listed in Table. It should be noted that also in this case the value of $D$ fulfils the condition $D \leq D_{0}$.

Figure $4 \mathrm{~A}$ shows the shape of the self-broadened Ar $750.3 \mathrm{~nm}$ line at pressure 0.79 Torr. In Fig. $4 \mathrm{~B}$ we plotted the weighted differences for the case when

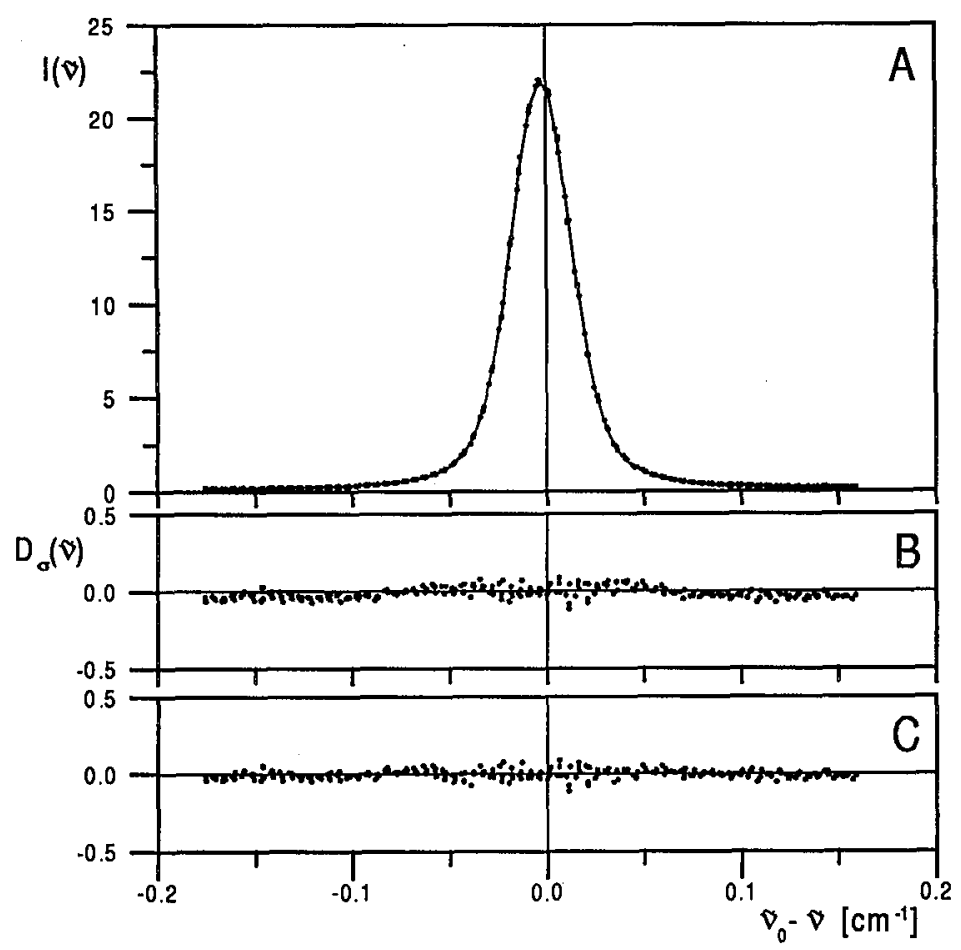

Fig. 4. (A) The shape $I(\tilde{\nu})$ (in relative units) of the self-broadened Ar $750.3 \mathrm{~nm}$ line at pressure 0.79 Torr. (B) The weighted differences $D_{\sigma}$ (in relative units) between the experimental and the best-fit value for $I_{\mathrm{fit}}(\widetilde{\nu})=I_{\mathrm{th}}(\widetilde{\nu})$ and $(\mathrm{C})$ for $I_{\mathrm{fit}}(\widetilde{\nu})=I(\widetilde{\nu})$. 
TABLE

The values of $\gamma_{\mathrm{DIS}}, E_{\mathrm{D}}$, and $D$ parameters determined for argon and neon lines; $D_{0}$ - dissociation energy [18].

\begin{tabular}{c|c|c|c|c|c}
\hline \hline$\lambda[\mathrm{nm}]$ & Emitter & $\gamma_{\text {DIS }}\left[10^{-3} \mathrm{~cm}^{-1}\right]$ & $E_{\mathrm{D}}[\mathrm{eV}]$ & $D[\mathrm{eV}]$ & $D_{0}[\mathrm{eV}]$ \\
\hline 748.8 & $\mathrm{Ne}$ & $124 \pm 17$ & $0.41 \pm 0.12$ & $1.12 \pm 0.12$ & $1.291 \pm 0.010$ \\
$753.5^{a}$ & & $110 \pm 15$ & $0.34 \pm 0.08$ & $1.20 \pm 0.08$ & \\
$754.4^{a}$ & & & & & \\
\hline 687.1 & $\mathrm{Ar}$ & $80 \pm 9$ & $0.28 \pm 0.06$ & $0.77 \pm 0.06$ & $1.320 \pm 0.005$ \\
703.0 & & $76 \pm 4$ & $0.27 \pm 0.03$ & $0.65 \pm 0.03$ & \\
\hline
\end{tabular}

${ }^{a}$ From paper [2].

$I_{\text {fit }}(\widetilde{\nu})=I_{\text {th }}(\widetilde{\nu})($ Eq. (4)). As is seen in this case we observe only very small departures as compared with those for the two previous Ar lines. Figure $4 \mathrm{C}$ shows the weighted differences for the case when $I_{\text {fit }}(\widetilde{\nu})=I(\widetilde{\nu})$ (Eq. (6)). As can be seen in this case the values of the weighted differences are only slightly more uniformly spread about zero than in case (B).

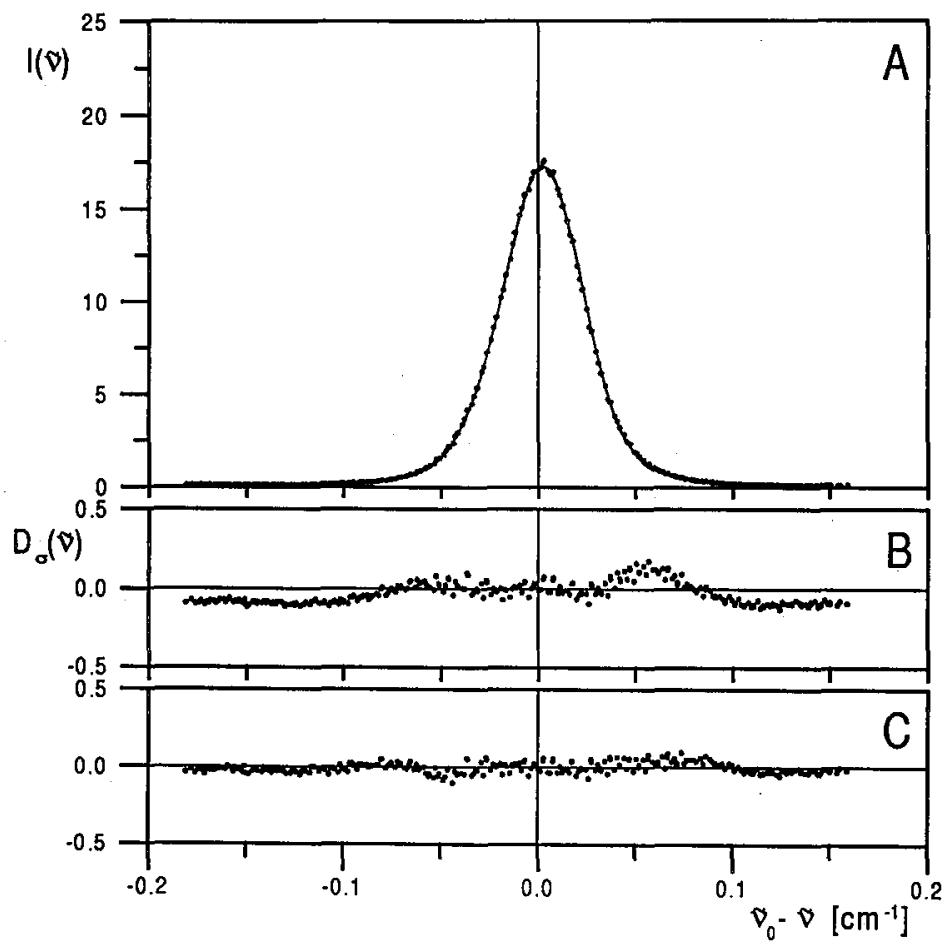

Fig. 5. (A) The shape $I(\widetilde{\nu})$ (in relative units) of the self-broadened Ne $748.8 \mathrm{~nm}$ line at pressure 1.57 Torr. (B) The weighted differences $D_{\sigma}$ (in relative units) between the experimental and the best fit value for $I_{\text {fit }}(\tilde{\nu})=I_{\text {th }}(\tilde{\nu})$ and $(C)$ for $I_{\text {fit }}(\widetilde{\nu})=I(\widetilde{\nu})$. 
Figure 5A shows the shape of the self-broadened Ne $748.8 \mathrm{~nm}$ line at pressure 1.57 Torr. From Figs. 5B and 5C we can see that the best quality of the fit is obtained for the $I(\widetilde{\nu})$ (Eq. (6)) profile.

Figure $6 \mathrm{~A}$ shows typical dependence of the best-fit values of the parameters $\kappa=N_{\text {nth }} / N_{\text {th }}$ decreasing with the neon pressure. The relation plotted in Fig. $6 \mathrm{~B}$ shows that the values of $\gamma_{\mathrm{DIS}}$ do not depend on the perturbing gas pressure. We determined $\gamma_{\mathrm{DIS}}, E_{\mathrm{D}}, D$, and their values are listed in Table. It should be noted that also for $\mathrm{Ne}^{*}-\mathrm{Ne}$ the value of $D$ fulfils the condition $D \leq D_{0}=$ $(1.291 \pm 0.010) \mathrm{eV}[18]$.

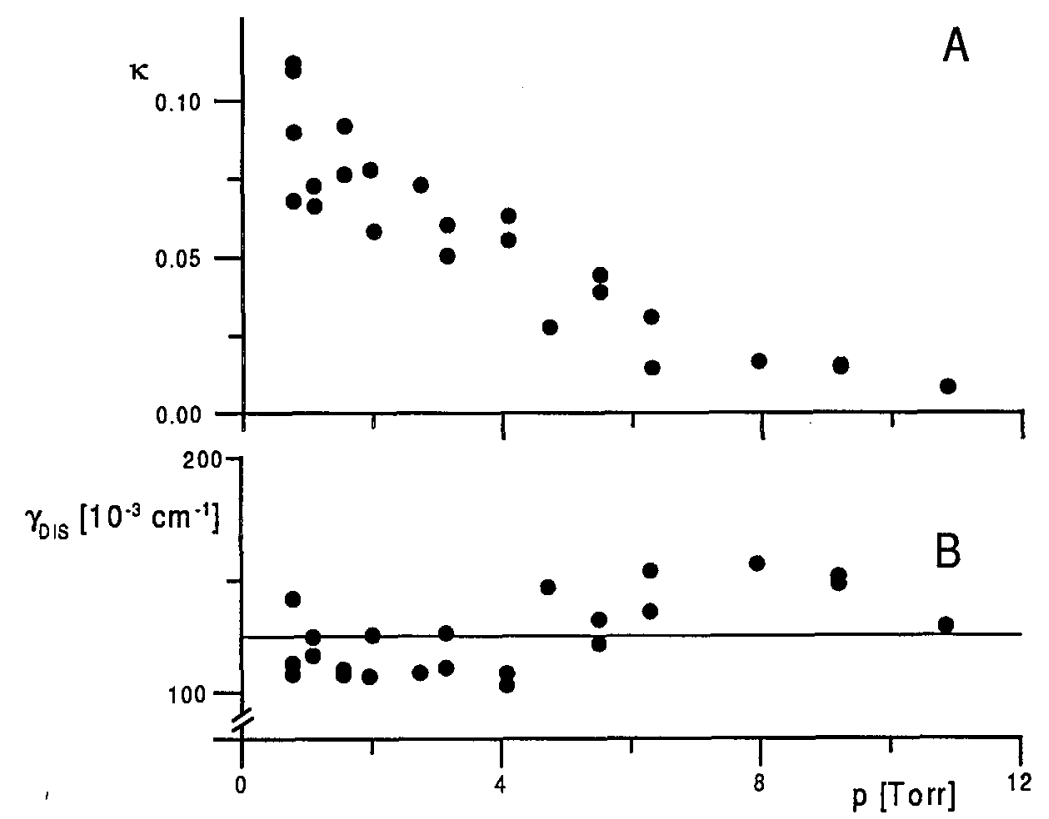

Fig. 6. The best-fit values of the parameters for Ne $748.8 \mathrm{~nm}$ line: (A) $\kappa=N_{\mathrm{nth}} / N_{\mathrm{th}}$ and (B) $\gamma_{D I S}$ plotted against the neon pressure.

Table shows the values of the $\gamma_{\mathrm{DIS}}, E_{\mathrm{D}}$, and $D$ determined for two argon lines and one neon line together with the values obtained for two neon lines in our earlier paper [2]. In the last column of this Table we quote the dissociation energies $D_{0}$ of molecular ions $\mathrm{Ar}_{2}^{+}$and $\mathrm{Ne}_{2}^{+}$in their ground electronic states ${ }^{2} \Sigma_{\mathrm{u}}^{+}$.

From Table we can see that the values of the kinetic energy in excess of the kinetic energy of the thermal motion $E_{\mathrm{D}}$ determined for the argon lines are lower than those for neon lines. It should be noted that, like in our earlier paper [2], in the present analysis we neglected the Gaussian component of the instrumental function of the Fabry-Perot interferometer. Accurate numerical tests performed recently $[6,7]$ have shown that the inclusion of this component does not influence the quality of the fit as well as does not make any essential change in the values of the parameters listed in Table. 


\section{Conclusions and summary}

In the present study we have shown that for 687.1 and $703.0 \mathrm{~nm}$ argon lines and 748.8 neon line emitted from the glow discharge at pressure about 1 Torr systematic departures of the registered line shape from the Voigt profile occur. We have also shown that these departures may be interpreted assuming that the dissociative recombination of $\mathrm{Ar}_{2}^{+}$ions is the main process responsible for the distortion of argon lines profiles. The similar departures caused by dissociative recombination of $\mathrm{Ne}_{2}^{+}$ions, reported for neon lines in paper [2], were confirmed for $748.8 \mathrm{~nm}$ neon line. It should be noted, however, that similar distortion of the line profile may be caused by any other process (e.g. [19]) additive to the thermal motion of atoms leading to the rectangular distribution of the excess speed.

\section{Acknowledgments}

The autors wish to express their gratitude to Professor J. Szudy for valuable help in the preparation of the manuscript.

This work was supported by a grant No. 673/PO3/96/10 (2 PO3B 005 10) from the Committee for Scientific Research.

\section{References}

[1] D.N. Stacey, R.C. Thompson, J. Phys. B 16, 537 (1983).

[2] R. Ciuryło, A. Bielski, J. Domysławska, J. Szudy, R.S. Trawiński, J. Phys. B, At. Mol. Opt. Phys. 27, 4181 (1994).

[3] L. Frommhold, M.A. Biondi, Phys. Rev. 185, 244 (1969).

[4] J. Wawrzyński, J. Wolnikowski, Phys. Scr. 33, 113 (1986).

[5] A. Bielski, J. Wawrzyński, J. Wolnikowski, Acta Phys. Pol. A 67, 621 (1985).

[6] R. Ciuryło, A. Bielski, S. Brym, J. Domysławska, D. Lisak, J. Szudy, R.S. Trawinski, Acta Phys. Pol. A 96, 359 (1999).

[7] A. Bielski, S. Brym, R. Ciuryło, J. Szudy, Eur. Phys. J. D, in press.

[8] T.R. Connor, M.A. Biondi, Phys. Rev. 140, A778 (1965).

[9] A.M. Biondi, Phys. Rev. 129, 1181 (1963).

[10] W.A. Rogers, M.A. Biondi, Phys. Rev. 134, A1215 (1964).

[11] J.A. Hornbeck, J.P. Molnar, Phys. Rev. 84, 625 (1951).

[12] S. Tolansky, High Resolution Spectroscopy, Methuen and Co., London 1947.

[13] E.A. Ballik, Appl. Opt. 5, 170 (1966).

[14] A. Bielski, J. Wolnikowski, Acta Phys. Pol. A 54, 601 (1978).

[15] A. Bielski, W. Dokurno, E. Lisicki, Z. Turło, Opt. Appl. 9, 151 (1981).

[16] D.W. Marquardt, J. Soc. Industr. Appl. Math. 11, 431 (1963).

[17] P.R. Berman, J. Quant. Spectrosc. Radiat. Transf. 12, 1331 (1972).

[18] R.I. Hall, Y. Lu, Y. Morioka, T. Matsui, T. Tanaka, H. Yoshii, T. Hayaishi, K. Ito, J. Phys. B, At. Mol. Opt. Phys. 28, 2435 (1995).

[19] J.F. Su, J.L. Nicol, J. Phys. B, At. Mol. Opt. Phys. 29, 4093 (1996). 